\title{
A shortest path approach to optic disc detection in retinal fundus
} images

Jeffrey Wigdahl, Pedro Guimaraes, Alfredo Ruggeri

Department of Information Engineering, University of Padova, Padova, Italy

\section{Abstract}

Aim: To evaluate a new algorithm to detect the optic disc in retinal fundus images on a number of publicly available datasets. Optic disc detection is an important first step in many automated algorithms, either to be masked out of future processing or for use in optic disc-related disease such as glaucoma and papilledema.

Methods: We propose a new method for optic disc detection that converts the retinal image into a graph and exploits vessel enhancement methods to calculate edge weights in finding the shortest path between pairs of points on the periphery of the image. The line segment with the maximum number of 'shortest paths' is considered the optic disc location, with refinement from a combination template matching approach in the found region. The method was tested on three publicly available datasets: DRIVE, DIARETDB1, and Messidor consisting of 40, 89, and 1200 images, respectively. All images were acquired at a $45^{\circ}-50^{\circ}$ field of view.

Results: The method achieves an accuracy of $100,98.88$, and $99.42 \%$ on the DRIVE, DIARETDB1, and Messidor databases, respectively.

Conclusions: The method performs as well or better than state-of-the-art methods on these datasets. Processing takes an average of 32 seconds $( \pm 1.2)$ to detect the optic disc, with 26 of those seconds used for the vessel enhancement process. The accuracy over a wide variety of images shows that the method is robust and would be optimal for retinal analysis systems that perform vessel enhancement as part of their processing. This would allow for optic disc and vessel segmentation to be performed simultaneously, saving processing time.

Keywords: graph theory, optic disc, retinal imaging, shortest path, template matching

Correspondence: Jeffrey Wigdahl, Department of Information Engineering, University of Padova, Via Gradenigo 6/B, 35131, Padova, Italy

E-mail:wigdahl@dei.unipd.it 


\section{Introduction}

Fundus imaging has been a staple in the clinical analysis of retinal diseases due to its simple, non-invasive visualization of the retinal vasculature. ${ }^{1}$ Studies have shown the correlation between changes in the retinal vasculature to vascular changes elsewhere in the body. ${ }^{2}$ This has made the screening and early detection of retinal diseases, such as hypertensive and diabetic retinopathies, an important and cost-effective method for preventing vision loss as well as monitoring systemic vascular disease. ${ }^{3}$ With the advancement of technology, automatic screening has become a feasible method for detecting disease and reducing the burden on the ophthalmologist. ${ }^{4}$ Automatic image processing and machine-learning techniques can detect, segment, and quantify retinal landmarks such as the retinal vasculature, fovea, and optic disc as well as lesions such as microaneurysms and exudates. ${ }^{5,6}$ Automated screening systems for diabetic retinopathy have already been deployed in certain countries that are able to flag early signs of disease so that these people can be seen by an ophthalmologist before the onset of vision loss.

The optic disc (OD) is one of the most important features of the retina and appears as a bright, yellowish oval. The size of the OD varies among adults with an average of 1.88 and $1.77 \mathrm{~mm}$ in the vertical and horizontal diameters. ${ }^{7}$ It is the entrance point for blood vessels into the retina and the exit point for ganglion cell axons, which form the optic nerve upon leaving the eye. Detection of the OD can aid in the detection of other retinal landmarks, such as the fovea and retinal arcades, which share geometric properties with the OD. ${ }^{8}$ Furthermore, analysis of the optic cup-to-disc ratio can aid in the diagnosis of glaucoma, ${ }^{9}$ while analysis of the OD boundary can be important for swelling of the OD in papilledema. ${ }^{10}$ Masking the OD out of the image can also be helpful as it removes a bright area with sharp contrast that could be falsely detected as vasculature or pathology.

This paper presents a new method for OD detection in retinal images that converts the retinal image to a graph and calculates the shortest path between selected points in the periphery of the image using edge weights calculated from a vessel enhanced image. Through the calculation of several shortest paths (using Dijkstra's shortest path algorithm), the algorithm is able to detect the vascular origin, and thus the OD location, after refinement using a combination template matching/ vertical edge detection image. Dijkstra's shortest path algorithm has been used in many image processing applications, ${ }^{11,12}$ but has not been used to our knowledge to detect the OD in this way. The method is evaluated on three publicly available datasets containing a total of 1329 images of varying levels of pathology and image quality, and has a combined detection rate of $99.40 \%$. These results show that the algorithm is robust and comparable with other state-of-the-art methods for OD detection.

The paper is organized in the following way. First, a literature review of recent OD detection methods is conducted, followed by a 'Methods' section which expands 
on the algorithm in more detail. The 'Results' section contains information on the datasets used for evaluation, examples from each dataset, and a comparison with other OD detection methods. This is followed by the 'Discussion' and 'Conclusions' sections.

\section{Literature review}

There have been many methods proposed in the literature for optic disc detection. Normally, the OD presents as the brightest region in the image. However, retinopathies, image quality, and normal variations can make this untrue, ${ }^{13}$ which makes OD localization a surprisingly difficult task that has led to many advanced methodologies. Some methods have focused just on OD detection, while others also perform OD segmentation. A look at many of the popular and newer OD detection algorithms will now be discussed. For an in-depth look at earlier OD algorithms, please see Youssif et al. for an extensive literature review. ${ }^{14}$ Hoover and Goldbaum used the fuzzy convergence of vessel end points from a vessel segmented image to determine the convergence of blood vessels and ultimately the OD location, obtaining an $89 \%$ detection rate on the STARE dataset..$^{15}$ Foracchia et al. fit a parabolic model to the main arcades in the image, with the OD location being the area of convergence of all vessels. ${ }^{16}$ Based on this model, the OD location is able to be determined even if it is not present in the image. The method achieved a $98 \%$ detection rate when tested on the STARE dataset. Neimeijer, Abramoff, and Van Ginneken used a k-nearest neighbor regressor (k-nearest neighbor regression is a regression technique that assigns an output value for the object in question based on the ' $k$ ' number of nearest training neighbors) with features from the original image and a vessel segmentation to predict the distance of each pixel in the image to the optic disc. ${ }^{17}$ The method achieved a $99.4 \%$ OD detection rate on an in-house dataset. Mahfouz et al. encoded the $x$ and $y$ coordinates of the OD in 1D projections based on certain retinal features. ${ }^{18}$ The method had a combined $97 \%$ detection rate on four publicly available datasets. Welfer et al. used an adaptive morphological approach that prunes a skeletonized vessel segmentation until just the main arcades are present. ${ }^{19}$ The OD is localized using the intersection of the centroid of the skeleton and the remaining vessel arcade fragment. The method obtained $100 \%$ and $97.75 \%$ detection rates on the DRIVE and DIARETDB1 databases. Aquino et al. combined three methods for OD detection (maximum difference, maximum variance, and a low-pass filtering method) into a voting procedure based on the location found in each method..$^{20}$ The method obtained a $99 \%$ detection rate in the Messidor database. Qureshi et al. also combined OD detection algorithms using geometric rules. ${ }^{21}$ The methods include pyramidal decomposition, edge detection, entropy, Hough transform, and feature vector combination, and achieved a $100 \%$ and $94.02 \%$ detection rate on the DRIVE and DIARETDB1 databases, respectively. 
Table 1. Comparison of methods that have reported results on one or more of the listed datasets

\begin{tabular}{|l|l|l|l|}
\hline OD Location Method & $\begin{array}{l}\text { DRIVE } \\
\text { dataset (\%) }\end{array}$ & $\begin{array}{l}\text { DIARETDB1 } \\
\text { dataset (\%) }\end{array}$ & $\begin{array}{l}\text { Messidor } \\
\text { dataset (\%) }\end{array}$ \\
\hline Sinthanayothin et al. ${ }^{1} 1999$ & 60 & - & - \\
\hline Walter et al. ${ }^{2}(2002)$ & 80 & - & - \\
\hline Youssif et al. ${ }^{14}(2008)$ & 100 & - & - \\
\hline Mahfouz et al. ${ }^{18}(2010)$ & 100 & 97.75 & - \\
\hline Welfer et al. ${ }^{19}(2010)$ & 100 & 97.75 & - \\
\hline Aquino et al. ${ }^{20}(2010)$ & - & - & 99 \\
\hline Yu et al. ${ }^{22}(2012)$ & - & - & 99.08 \\
\hline Zubair et al. ${ }^{3}(2013)$ & - & - & 98.65 \\
\hline Saleh et al. ${ }^{4}(2014)$ & 100 & - & - \\
\hline Yu, Ma, \& ii $^{24}(2015)$ & 100 & 98.88 & 99.67 \\
\hline Abdullah et al. ${ }^{26}(2016)$ & 100 & 100 & 99.25 \\
\hline Proposed Method & 100 & 98.88 & 99.42 \\
\hline
\end{tabular}

Yu et al. used adaptive template matching to determine OD candidates and the final OD location was determined from the vessel characteristics in these regions. ${ }^{22}$ The method achieved a $99.08 \%$ detection rate on the Messidor dataset. Pereira et al. used an ant colony optimization algorithm which mimics swarm behavior as the ants move based on local intensity variation, achieving a 93.25\% detection rate on the DIARETDB1 database. ${ }^{23} \mathrm{Yu}, \mathrm{Ma}$, and Li employed a morphological approach on a combination of intensity and vessel convergence information and achieved a $99.52 \%$ detection rate on several publicly available datasets. ${ }^{24}$ Rahebi et al. detected the OD location using the firefly algorithm. ${ }^{25}$ The algorithm compares fireflies, moving the insects based on intensity values in the image until an optimal insect is found as the OD location. The method achieved a $100 \%$ and $94.38 \%$ detection rate in the DRIVE and DIARETDB1 databases, respectively. Abdullah et al. used the circular Hough transform after morphologically removing the vessels from a preprocessed image to determine the OD location. ${ }^{26}$ Results for many of these methods are summarized in Table 1.

\section{Methods}

This work calculates the shortest path between selected points on the periphery of a fundus image, based on edge weights calculated from a vessel enhanced image, to determine the approximate OD location. The segment with the maximum number 
of 'shortest paths' is combined with the results from OD template matching and the vertical Hessian component to localize the OD location. Correlation values are then used as a check to determine if the location found is a likely OD location. If not, an iterative process checks larger portions of the shortest path until conditions are satisfied; if not, a lower bound reverts the location back to the original. Figure 1 shows the flow of the algorithm from preprocessing through OD localization.

\subsection{Preprocessing}

All preprocessing steps are performed for vessel enhancement. Images are resized based on image resolution and the background is cropped before processing. All processing is performed on the green channel image due to its superior contrast between blood elements and background. To prevent unwanted contrast along the circular edge of the field of view, a mirroring technique is used to fill in the remaining black background pixels. A mask, $G_{\text {MASK }}$, of the field of view is created, with all pixels beyond the mask needing to be mirrored. Pixel values are mirrored from the original green channel image, $G$, based on their distance from the circular edge of $G_{\text {MASK }}$ and the angle with respect to the center of $G_{\text {MASK }}$. This removes all the black background pixels and leaves a rectangular region for future processing. The image then undergoes illumination correction by means of background estimation. The background illumination is estimated using a Gaussian low-pass filter with a large kernel, as in the following equation:

$$
G_{\text {ill }}=G(x, y)-G_{L P F}(x, y)+\operatorname{mean}\left(G_{L P F}(x, y)\right)
$$

where $G_{L P F}$, the low-pass filtered version of $G$, is subtracted from $G$ and the mean value of $G_{L P F}$ is then added back to create $G_{\text {ill, }}$, the illumination-corrected image. The largest kernel size used for the low-pass filter is $1 / 10^{*}(($ rows + columns $) / 2)$. The contrast was then normalized and enhanced using contrast limited adaptive histogram equalization (CLAHE), which operates on windows of pixels rather than attempting to equalize the histogram of the entire image at once. ${ }^{27}$

\subsection{Edge weight calculation}

An undirected graph $\operatorname{Gr}(V, E)$ is a set of vertices and edges, where in our images $V$ is every pixel in the foreground of the image and $E$ is the set of connections between pixels. Each edge, $E$, can have an associated weight. General values for edge weights can be some measure of distance between vertices or, in this case, the images' pixel intensity values. The edge weights are taken as the pixel values after performing Frangi vessel enhancement. ${ }^{28}$ As a short explanation, Frangi vessel enhancement uses the eigenvalues of the Hessian 2D matrix of the image to enhance vessel-like structures. The Hessian consists of second-order partial derivatives, as shown in Equation (2), which are generally smoothed by a simple Gaussian function: 


$$
H(x, y)=\left|\begin{array}{ll}
D x x & D x y \\
D y x & D Y Y
\end{array}\right|
$$

where $D_{x x}$ through $D_{y y}$ are the partial second derivatives of the Hessian, $H(x, y)$, that describe the gradient of the image in different directions. The amount of smoothing is controlled by the standard deviation (sigma) of the Gaussian function. From the Hessian, the eigenvalues are decomposed and the following formulas are used to extract candidate tubular information:

$$
\begin{aligned}
& R_{B}=\left(\frac{\lambda_{1}}{\lambda_{2}}\right)^{2} \\
& S=\sqrt{\left(\lambda_{1}\right)^{2}+\left(\lambda_{2}\right)^{2}}
\end{aligned}
$$

where $\lambda_{1}$ and $\lambda_{2}$ are the two largest eigenvalues, $R_{B}$ represents a 'blobness' measure, and $S$ represents a second-order structure measure. These two metrics are then combined into the following 'vesselness' metric:

$$
I_{\text {enh }}(S)=\left\{\begin{array}{cc}
0 & \text { if } \lambda_{2}<0 \\
\exp \left(-\frac{R_{\mathrm{B} 2}}{2 \beta^{z}}\right) & \left(1-\left(-\frac{S^{z}}{2 c^{z}}\right)\right)
\end{array} \mid\right.
$$

where $\beta$ and $c$ are constants that control the tubular filter. $I_{\text {enh }}(s)$ is the vessel enhanced image at sigma value $s$. Enhancement is performed at multiple scales (varying the standard deviation of the Gaussian smoothing filter), and the maximum response at each pixel becomes the vessel enhanced image. In this particular case, we want to ignore the smaller vessels and only capture the primary and secondary vessels in the image. Thus, a higher band of sigma values (16-26) was used so that the tertiary vessels were not enhanced. The values from the vessel enhanced image are used as the edge weights in our graph after being normalized between 0 and 255, and inverted so that vessel pixels have lower weights. We must now determine the starting and ending points from which to calculate the shortest paths. The chosen points are equally spread around the periphery of the graph. The image is cut into four quadrants, as shown in Figure 1. Starting points in quadrants 1 and 2 are matched with end points in quadrants 3 and 4 . Four points from each quadrant are matched with each point in the adjacent quadrant for a total of 32 shortest paths. As converting the image to a graph and calculating the shortest paths is an expensive process, the image is down-sampled by a factor of four after vessel enhancement. To calculate the shortest path between the selected points, Dijkstra's shortest path algorithm is used. ${ }^{29}$ The algorithm allows for the finding of the shortest path from a starting node to every other node in the graph in $O\left(\log (V)^{\star} E\right)$ time, where $V$ is the number of vertices and $E$ is the number of edges. In the attempt to find the shortest 

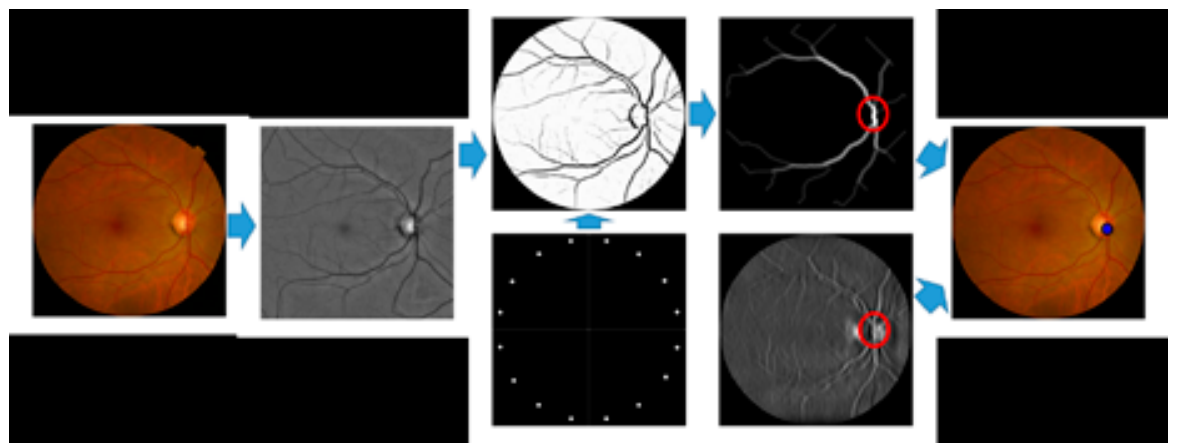

Fig. 1. Example of algorithm flow. The original image is cropped, mirrored, and the illumination is corrected. The Frangi vessel-enhanced image is used for the graph weights. Points along the outside of the image are connected via shortest path across quadrants. The area with the maximum number of 'shortest paths' is the initial search zone, and the final optic disc location comes as the maximum of the edge detection/template matching combination on that line.

path to the other side of the image, the main arcades and secondary vessels will act as highways, directing the shortest path through the convergence of vessels at the optic disc. As more 'shortest paths' are calculated, a histogram is built up of the number of shortest paths at each point in the graph, corresponding to each pixel in the image. The maximum line segment from the histogram will be the rough estimate of the OD location.

\subsection{Template matching/edge detection}

The use of OD templates and vertical vessel information has been used before to detect the OD and has proven effective in its own right. ${ }^{22}$ In this case, the method differs in that the information is combined into a single image and used only in the line segment of the OD candidate. The line segment found from the shortest path must be narrowed to a single pixel location. To do this, a combination of correlation with an OD template and vertical edge information is calculated in the region of interest. The OD template incorporates the assumption that the OD is a bright oval region with darker vessels exiting nasally, while the vertical edge information assumes that the main arcades exit the OD vertically before curving into a parabolic shape. The calculation of the vertical edge information has already been carried out when we previously calculated the Hessian for vessel enhancement.

We use the normalized gradient information from $D_{y y}$ in Equation (2) as the vertical vessel component combined with template matching correlation scores. The OD template used is $100 \times 100$ pixels and is composed of a combination of ten randomly selected ODs extracted from ten images from an in-house dataset, averaged together and normalized. Since there is no a priori information or recognition of whether the image is from a right or left eye, two templates were used. The original was created 
from left-eye images and then flipped for right eyes, as using a different template for both eyes could create bias. The maximum value combining the normalized template correlation values and vertical gradient information along the segment with the highest number of shortest paths gives the OD location.

\subsection{Correction}

There are several cases where it becomes clear the incorrect OD location has been found. While this happens rarely, an iterative process is deployed to determine if there is a more likely OD location. If the template/vessel gradient information is much lower than the maximum value in the image, the maximum segment requirement is decreased to allow for a slightly larger search area. This process will continue until areas that have $60 \%$ of the maximum number of shortest paths have been checked. At this point, if a better candidate has not been found, the OD location will revert to the original location found on the segment with the maximum number of shortest paths.

\section{Results}

Three publicly available datasets were used to evaluate the algorithm. These datasets combine varying levels of disease and image quality. The OD boundary was manually determined except for the Messidor dataset, which used the optic

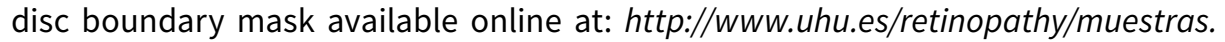
php. ${ }^{20}$ For all datasets, a correct OD detection was any point found within the OD boundary.

\subsection{Datasets}

DRIVE: The DRIVE dataset was created to compare vessel segmentation algorithms and consists of 40 images ( 7 with mild diabetic retinopathy, 33 with no signs of disease) used to make clinical diagnoses. The images were acquired on a Canon CR5 non-mydriatic camera at $768 \times 584$ pixels and a $45^{\circ}$ field of view, and were compressed into JPEG. ${ }^{30}$ While this dataset consists of high-quality images with little disease, it has been used to benchmark OD detection algorithms for many years and is included for this reason.

DIARETDB1: The diabetic retinopathy database and evaluation protocol consists of 89 images ( 5 with no signs of disease, 84 with at least mild non-proliferative retinopathy). Images were acquired at $1152 \times 1500$ pixels and a $50^{\circ}$ field of view, and saved in PNG format. ${ }^{31}$

Messidor: The Messidor database consists of 1200 images, 800 with pupil dilation and 400 without. All images were acquired using a Topcon TRC NW6 non-mydriatic 
camera at a $45^{\circ}$ field of view. The images were captured using 8 bits per color plane at $1440 \times 960,2240 \times 1488$, or $2304 \times 1536$ pixels. $^{32}$

\subsection{OD detection}

Results are given for the three publicly available datasets and compared with many other algorithms on these datasets which can be found in Table 1. The results for the DRIVE dataset obtained a 100\% detection rate of the optic disc. Table 1 shows that many methods obtain these same results, as these images are typically of good quality and contain very little disease (seven images with mild diabetic retinopathy). It should also be noted that correction in this dataset was unnecessary. Figure 2 shows a sampling of correctly detected images from the database. A $98.88 \%$ detection rate was achieved on the DIARETDB1 database, which amounts to missing a single image from the 89-image dataset; this image is the last one in Figure 5. A combination of a dark OD and low contrast in the area had the algorithm second-guess its initial estimate, which was just below the OD. Correctly identified OD locations from the dataset can be seen in Figure 3. The dataset contains 84 images with at least mild diabetic retinopathy. Since nothing in this algorithm depresses the contribution of lesions in the vessel enhancement step, large numbers of lesions can occasionally create a short circuit in the shortest paths calculation, splitting some paths and requiring correction to properly detect the OD. Even though this occurred, the algorithm was able to correct the OD location in each case. The optic disc was found in 1193 of the 1200 images in the Messidor data set for a $99.42 \%$ detection rate. Correctly detected OD locations for this database can be seen in Figure 4. All seven of the misses can be seen in Figure 5. The main reason for incorrect detection was blur, either from poor image quality or possible cataract that covered either the OD vessels or the main arcades from an entire hemisphere of the image. The second reason for misses was bright regions near or around the OD. These non-OD bright regions will have edges that appear in the vessel enhancement. Figure 6 shows

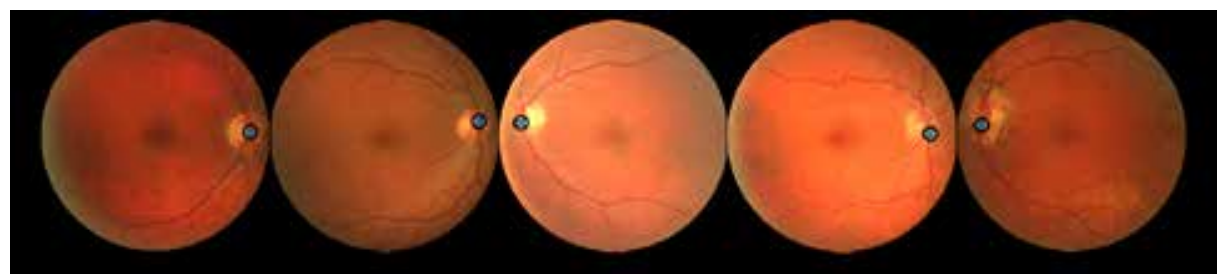

Fig. 2. Sampling of correctly detected images from the DRIVE database. Estimated OD location is marked with a cross surrounded by a circle. 


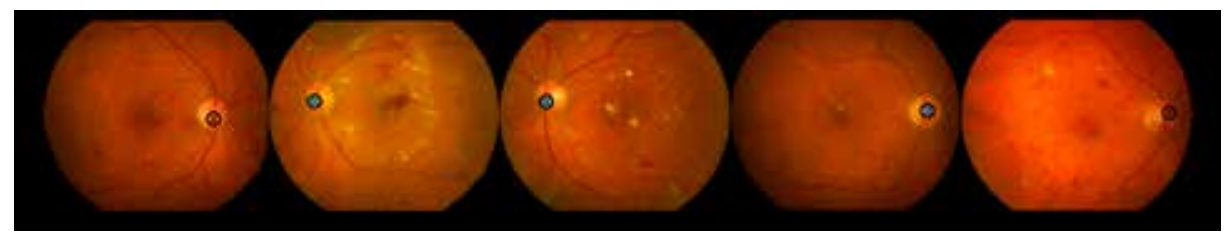

Fig. 3. Correctly identified OD location in images from the DIARETDB1 database.

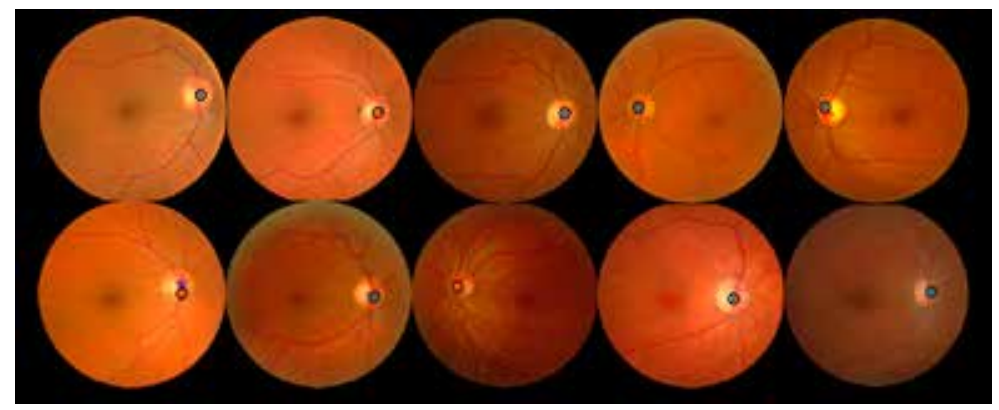

Fig. 4. Correctly detected OD location in images from the Messidor database.

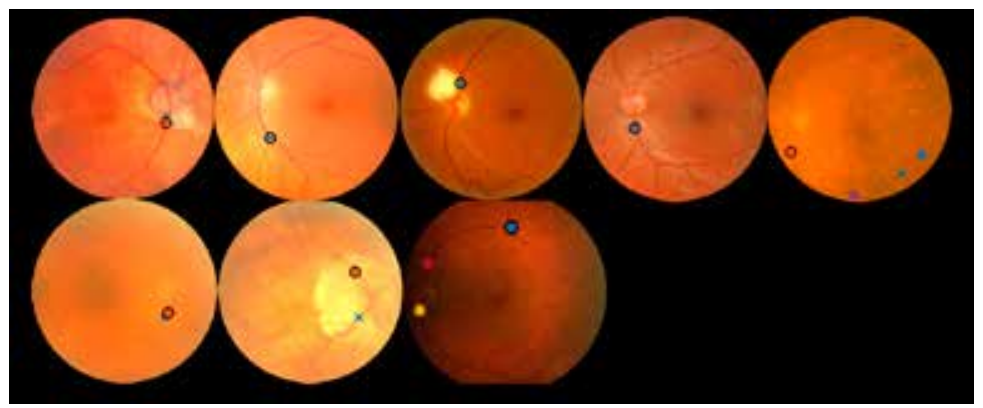

Fig. 5. All incorrectly detected images. (1-7) Messidor. (8) DIARETDB1. Final estimated OD location is a cross with a circle around it. Attempts at correction are crosses or stars that have not been circled.

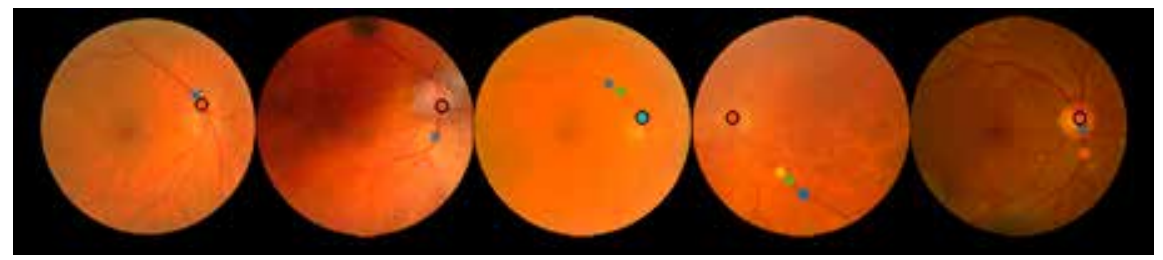

Fig. 6. Incorrect OD locations that where corrected due to the low likelihood of the original location being the true OD by the iterative correction of the algorithm. 
examples of the iterative corrective process successfully finding the correct OD after being initially incorrect.

\section{Discussion}

The algorithm was implemented in Matlab 2016a on a desktop computer (Intel i7 CPU at $3.4 \mathrm{GHz}$ and $16 \mathrm{~Gb}$ RAM), and average run time was $32 \pm 1.2$ seconds per image. This time held relatively constant through each dataset due to the up-sampling of the images prior to processing. This could be greatly reduced through code optimization and choice of programming language. Also, 26 of those seconds were spent calculating the Frangi vessel enhanced image to calculate the edge weights for the graph. The Frangi method is not the only one that produces a vessel enhanced image, and other methods could replace this one in order to reduce running time or improve results. The number of points used to calculate shortest paths can also affect the results. The number chosen for this study (four per quadrant) was based on experimentation as a tradeoff between results and processing time. More points could shrink the initial search region for the OD, but placing points too close together will only raise processing time as these points will likely choose the same shortest path if they are all near the same vessel at the periphery.

This idea presents a global approach to vessel convergence and relies heavily on the presence of the main arcades being present in the image. This does not usually occur in images where the field of view is less than $45^{\circ}$. Older datasets used for testing, such as the STARE dataset, ${ }^{15}$ which was widely used to validate OD detection methods, contains many images at a $30^{\circ}$ field of view or less. Since there is no convergence of vessels without the main arcades, the algorithm was not benchmarked on this dataset. However, of the 81 images in the STARE dataset, 46 of the images meet the vessel convergence criteria as either having the main arcades present or being OD-centered images, in which vessel convergence is assured. In this subset, the algorithm was unable to detect the OD in four images for a $91.3 \%$ detection rate.

\section{Conclusions}

This work presents a novel method for OD localization based on the shortest path between points on the periphery of the image using edge weights calculated from a vessel enhanced image to detect the likely convergence of vessels at the OD. The algorithm achieves results comparable to or better than results from state-of-theart algorithms on the selected databases. The excellent results achieved from the Messidor database show that this method would be useful in an automated screening system or retinal vessel analysis system, where the processing time taken 
for vessel enhancement can be used both for OD detection and vessel segmentation, saving overall processing time.

\section{Acknowledgements}

This research was made possible by a Marie Curie grant from the European Commission within the Retinal Vascular Modeling, Measurement and Diagnosis Initial Training Research Network (REVAMMAD ITN) framework, Project number 316990.

\section{References}

1. Jack JK, Brad B. 2015. Clinical ophthalmology: a systematic approach. London: Elsevier Health Sciences.

2. Witt N, Wong TY, Hughes AD, Chaturvedi N, Klein BE, Evans R, McNamara M, Thom SA, Klein R: Abnormalities of retinal microvascular structure and risk of mortality from ischemic heart disease and stroke. Hypertension 47:975-981, 2006 DOI: 10.1161/01.HYP.0000216717.72048.6c

3. James M, Turner DA, Broadbent DM, Vora J, Harding SP. Cost effectiveness analysis of screening for sight threatening diabetic eye disease. BMJ 2000;320:1627-31 PMID:10856062

4. Philip S., Fleming A. D., Goatman K. A., Fonseca S., Mcnamee P., Scotland G. S., Prescott G. J., Sharp P. F., and Olson J. A., The efficacy of automated "disease/no disease" grading for diabetic retinopathy in a systematic screening programme, Br. J. Ophthalmol., vol. 91, pp. 1512-1517, 2007.

5. Fleming A. D., Philip S., Goatman K. A., Williams G. J., Olson J. A, and Sharp P. F., Automated detection of exudates for diabetic retinopathy screening, Phys. Med. Biol., vol. 52, pp. 7385-7396, 2007.

6. Niemeijer M., van Ginneken B., Staal J., Suttorp-Schulten M. S. A., and Abràmoff M. D., Automatic detection of red lesions in digital color fundus photographs, IEEE Trans. Med. Imag., vol. 24, no. 5, pp. 584-592, May 2005.

7. Quigley HA, Brown AE, Morrison JD, Drance SM: The size and shape of the optic disk in normal human eyes. Arch Ophthalmol 108:51-57, 1990.

8. Welfer D, Scharcanski J, Marinho D. Fovea center detection based on the retina anatomy and mathematical morphology. Comput Methods Programs Biomed 2011;104(3):397-409.

9. Abramoff MD, Garvin MK, Sonka M. 2010. Retinal imaging and image analysis. IEEE Reviews in Biomedical Engineering 3:169_208 DOI 10.1109/RBME.2010.2084567.

10. 10. Echegaray S, Zamora G, Yu H, Luo W, Soliz P, Kardon R (2011) Automated analysis of optic nerve images for detection and staging of papilledema. Invest Ophthalmol Vis Sci 52:7470-7478.

11. Joshi V. S., Garvin M. K., Reinhardt J. M., and Abramoff M. D., Automated method for the identification and analysis of vascular tree structures in retinal vessel network, inProc. SPIE Conf. Med. Imag., 2011, vol. 7963, no. 1, pp. 1-11.

12. Merickel M. B., Abramoff M. D., Sonka M., and Wu X., Segmentation of the optic nerve head combining pixel classification and graph search, Proc. SPIE, p. 651215-10, 2007.

13. Haleem MS, Han L, Van Hemert J, Li B. 2013. Automatic extraction of retinal features from colour retinal images for glaucoma diagnosis: a review. Computerized Medical Imaging and Graphics 37:581_596 DOI 10.1016/j.compmedimag.2013.09.005. 
14. Youssif AA-HA-R, Ghalwash AZ, Ghoneim AASA-R. 2008. Optic disc detection from normalized digital fundus images by means of a vessels' direction matched filter. IEEE Transactions on Medical Imaging 27:11_18 DOI 10.1109/TMI.2007.900326.

15. Hoover A, Goldbaum M. 2003. Locating the optic nerve in a retinal image using the fuzzy convergence of the blood vessels. IEEE Transactions on Medical Imaging 22:951_958 DOI 10.1109/ TMI.2003.815900.

16. Foracchia M, Grisam E, Ruggeri A (2004) Detection of optic disc in retinal images by means of a geometrical model of vessel structure. IEEE Trans Med Imaging 23:1189-1195.

17. Niemeijer M, Abràmoff MD, Van Ginneken B. 2009. Fast detection of the optic disc and fovea in color fundus photographs. Medical Image Analysis 13:859_870 DOI 10.1016/j.media.2009.08.003.

18. Mahfouz AE, Fahmy AS. 2010. Fast localization of the optic disc using projection of image features. IEEE Transactions on Image Processing 19:3285_3289 DOI 10.1109/TIP.2010.2052280.

19. Welfer D, Scharcanski J, Kitamura CM, Dal Pizzol MM, Ludwig LW, Marinho DR. 2010. Segmentation of the optic disk in color eye fundus images using an adaptive morphological approach. Computers in Biology and Medicine 40:124_137 DOI 10.1016/j.compbiomed.2009.11.009.

20. Aquino A, Gegúndez-Arias ME, Marín D. 2010. Detecting the optic disc boundary in digital fundus images using morphological, edge detection, and feature extraction techniques. IEEE Transactions on Medical Imaging 29:1860_1869 DOI 10.1109/TMI.2010.2053042.

21. Qureshi RJ, Kovacs L, Harangi B, Nagy B, Peto T, Hajdu A (2012) Combining algorithms for automatic detection of optic disc and macula in fundus images. Comput Vis Image Underst 116:138-145

22. Yu H, Barriga ES, Agurto C, Echegaray S, Pattichis MS, Bauman W, Soliz P. 2012. Fast localization and segmentation of optic disk in retinal images using directional matched filtering and level sets. IEEE Transactions on Information Technology in Biomedicine 16:644_657 DOI 10.1109/ TITB.2012.2198668.

23. Pereira C, Gonçalves L, Ferreira M (2013) Optic disc detection in color fundus images using ant colony optimization. Med Biol Eng Comput 51:295-303

24. Yu T, Ma Y, Li W. 2015. Automatic localization and segmentation of optic disc in fundus image using morphology and level set. In: Medical information and communication technology (ISMICT), 2015 9th international symposium on. IEEE, 195_199.

25. Rahebi, J. \& Hardalaç, F. (2016) A new approach to optic disc detection in human retinal images using the firefly algorithm. Med Biol Eng Comput 54: 453. DOI 10.1007/s11517-015- 1330-7.

26. Abdullah et al. (2016), Localization and segmentation of optic disc in retinal images using circular Hough transform and grow-cut algorithm. PeerJ 4:e2003; DOI 10.7717/peerj.2003

27. Zuiderveld K., (1994) Contrast limited adaptive histogram equalization, in Graphics Gems IV, P. S. Heckbert, Ed., pp. 474-485, Academic Press, Boston, Mass, USA.

28. Frangi, A.F., Niessen, W.J., Vincken, K.L., Viergever, M.A., 1998. Multiscale vessel enhancement filtering. In: Proc. Med. Image Comput. Assist. Interv., vol. 1496, pp. 130-137.

29. Dijkstra, E. W. (1959). A note on two problems in connexion with graphs. Numerische Mathematik. 1: 269-271. doi:10.1007/BF01386390.

30. Staal J, Abramoff MD, Niemeijer M, Viergever MA, Ginneken BV. 2004. Ridge-based vessel segmentation in color images of the retina. IEEE Transactions on Medical Imaging 23:501_509 DOI 10.1109/ TMI.2004.825627.

31. Kauppi T, Kalesnykiene V, Kamarainen J-K, Lensu L, Sorri I, Raninen A, Voutilainen R, Pietilä J, Kälviäinen H, Uusitalo H. 2007. DIARETDB1 diabetic retinopathy database and evaluation protocol. Medical Image Understanding and Analysis 2007:61 DOI 10.5244/C.21.15.

32. Decenciere E, Zhang X, Cazuguel G, La, B, Cochener B, Trone C, Gain P, Ordónez-Varela J-R, Massin P, Erginay A. 2014. Feedback on a publicly distributed image database: the Messidor database. Image Analysis and Stereology 33(3):231_234 DOI 10.5566/ias.1155.

33. Sinthanayothin C, Boyce JF, Cook HL, Williamson TH (1999) Automated localization of the optic disc, fovea, and retinal blood vessels from digital color fundus images. $\mathrm{Br} \mathrm{J}$ Ophthalmol 83:902-910. 
34. Walter T, Klein JC, Massin P, Erginary A (2002) A contribution of image processing to the diagnosis of diabetic retinopathy-detection of exudates in color fundus images of human retina. IEEE Trans Med Imaging 21:1236-1243.

35. Zubair M, Yamin A, Khan SA. 2013. Automated detection of optic disc for the analysis of retina using color fundus image. In: Imaging systems and techniques (IST), 2013 IEEE international conference on. Piscataway: IEEE, 239-242.

36. Saleh MD, Salih ND, Eswaran C, Abdullah J. 2014. Automated segmentation of optic disc in fundus images. In: 2014 IEEE 10th International Colloquium on signal processing \& its applications (CSPA). Piscataway: IEEE, 145-150. 